PREPARED FOR THE U.S. DEPARTMENT OF ENERGY, UNDER CONTRACT DE-AC02-76CH03073

PPPL-4000

PPPL-4000

UC-70

\author{
Microturbulent Drift Mode Stability \\ before Internal Transport Barrier Formation \\ in the Alcator C-Mod Radio Frequency Heated H-mode
}

by

M.H. Redi, W. Dorland, C.L. Fiore, P.T. Bonoli, M.J. Greenwald,

J.E. Rice, J.A. Baumgaertel, T.S. Hahm, G.W. Hammett,

K. Hill, D.C. McCune, D.R. Mikkelsen, and G. Rewoldt

August 2004

NM|

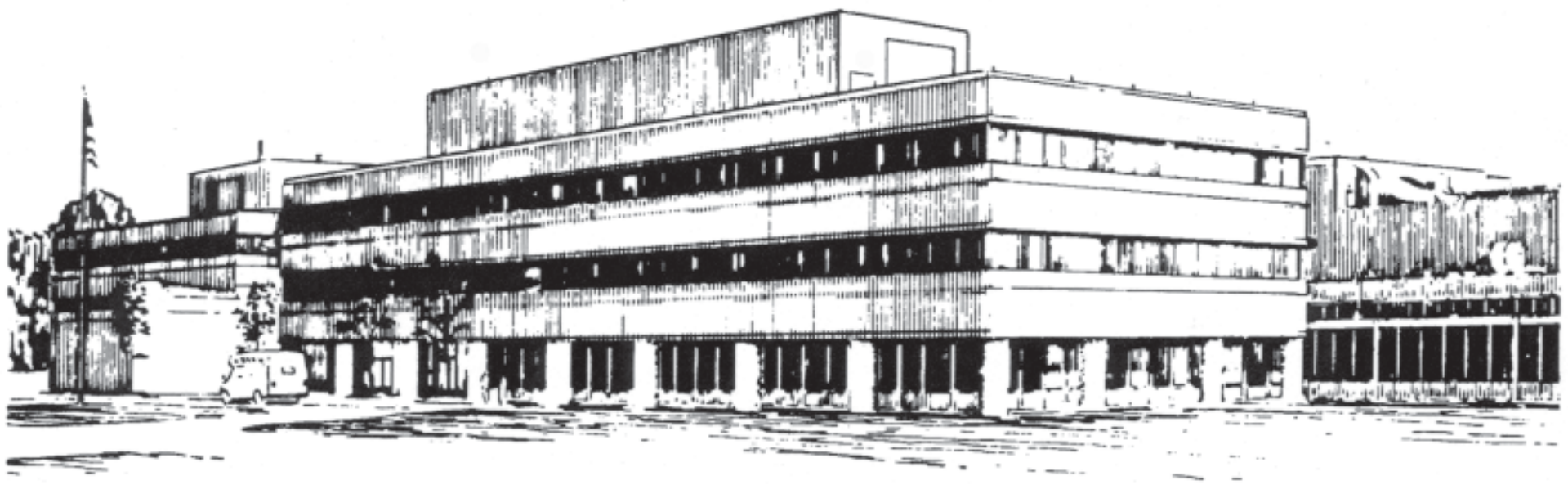

PRINCETON PLASMA PHYSICS LABORATORY PRINCETON UNIVERSITY, PRINCETON, NEW JERSEY 


\section{PPPL Reports Disclaimer}

This report was prepared as an account of work sponsored by an agency of the United States Government. Neither the United States Government nor any agency thereof, nor any of their employees, makes any warranty, express or implied, or assumes any legal liability or responsibility for the accuracy, completeness, or usefulness of any information, apparatus, product, or process disclosed, or represents that its use would not infringe privately owned rights. Reference herein to any specific commercial product, process, or service by trade name, trademark, manufacturer, or otherwise, does not necessarily constitute or imply its endorsement, recommendation, or favoring by the United States Government or any agency thereof. The views and opinions of authors expressed herein do not necessarily state or reflect those of the United States Government or any agency thereof.

\section{Availability}

This report is posted on the U.S. Department of Energy's Princeton Plasma Physics Laboratory Publications and Reports web site in Fiscal Year 2004. The home page for PPPL Reports and Publications is: http://www.pppl.gov/pub_report/

DOE and DOE Contractors can obtain copies of this report from:

U.S. Department of Energy

Office of Scientific and Technical Information

DOE Technical Information Services (DTIS)

P.O. Box 62

Oak Ridge, TN 37831

Telephone: (865) 576-8401

Fax: (865) 576-5728

Email: reports@adonis.osti.gov

This report is available to the general public from:

National Technical Information Service

U.S. Department of Commerce

5285 Port Royal Road

Springfield, VA 22161

Telephone: $1-800-553-6847$ or

(703) $605-6000$

Fax: (703) 321-8547

Internet: http://www.ntis.gov/ordering.htm 


\title{
Microturbulent drift mode stability before internal transport barrier formation in the Alcator C-Mod radio frequency heated H-mode
}

\author{
M. H. Redi \\ Princeton Plasma Physics Laboratory, Princeton, NJ 08543, USA \\ W. Dorland \\ Institute for Plasma Research, U. MD, College Park, MD 20742, USA \\ C. L. Fiore, P. T. Bonoli, M. J. Greenwald, J. E. Rice \\ Plasma Science and Fusion Center, MIT, Cambridge, MA 02139, USA \\ J. A. Baumgaertel, T. S. Hahm, G. W. Hammett, K. Hill, D. C. McCune, \\ D. R. Mikkelsen, G. Rewoldt \\ Princeton Plasma Physics Laboratory, Princeton, NJ 08543, USA
}

(Received

H-mode experiments on Alcator C-Mod [I. H. Hutchinson, et al., Phys. Plas. 1, 1511 (1994)] which exhibit an internal transport barrier (ITB), have been examined with gyrokinetic simulations, near the ITB onset time. Linear simulations support the picture of ion and electron temperature gradient (ITG, ETG) microturbulence driving high $\square_{i}$ and $\square_{e}$, respectively, and that stable ITG correlates with reduced particle transport and improved ion thermal confinement on C-Mod. In the barrier region ITG is weakly unstable, with a critical temperature gradient higher than expected from standard models. Nonlinear calculations and the role of $\boldsymbol{E} \boldsymbol{x} \boldsymbol{B}$ shear suppression of turbulence outside the plasma core are discussed in light of recent profile measurements for the toroidal velocity. The gyrokinetic model benchmarks successfully against experiment in the plasma core.

(PACS numbers: 52.30.Gz, 52.35.Ra, 52.55.F 


\section{INTRODUCTION}

When internal barriers to plasma particle and energy transport develop, high energy plasma is well confined, a necessary step toward economical fusion reactors. Because Alcator C$\operatorname{Mod}^{1}$ is a toroidal magnetic confinement device with high toroidal field, high plasma density, and radio frequency (RF) heating, its transport characteristics are of special interest, being relevant to fusion reactor scenarios ${ }^{2}$. In this paper, plasma conditions just before internal transport barrier (ITB) formation on C-Mod are analysed for drift mode microinstability using the GS2 $2^{3,4}$ gyrokinetic code. Through consideration of plasma stability just before the ITB, we seek to identify those conditions most optimal and detrimental to ITB formation. The massively parallel simulations are fully electromagnetic, following four plasma species and include the complete electron response. The internal transport barrier (ITB) experiment under study is the

subject of much recent analysis ${ }^{5-12}$, including Ref. 12 which reports gyrokinetic simulations of strong trapped electron mode (TEM) microturbulence late in the discharge, during the fully developed ITB phase. We combine velocity profile data ${ }^{13}$, transport analysis and the linear growth rates at the ITB onset time to integrate data and simulation and explore the effects of toroidal velocity measurements on benchmarking simulations. The paper is organized as follows: the experimental conditions are presented in Sec. II. In Sec. III the simulations and results are described, followed by a discussion in Sec. IV. We conclude with a summary of the results in Sec. V.

\section{ALCATOR C-MOD INTERNAL TRANSPORT BARRIER EXPERIMENT}

Following off-axis RF heating and establishment of an H-mode, C-Mod develops a reproducible ITB with a very steep electron density profile (see Figs. 1-2). Ion and electron thermal confinement are found from experimental analysis to be better than neoclassically 
predicted in the plasma core. A typical such experiment is analysed here, pulse \#1001220016. It is characterized by $R_{o}=0.69 \mathrm{~m}, B_{o}=4.5 \mathrm{~T}, I p=0.78 \mathrm{MA}$, and central electron density $=$ $4 \times 10^{20} / \mathrm{m}^{3}$. Deuterium majority ion and impurities of oxygen, carbon, boron and molybdenum give $<Z_{\text {eff }}>\sim 1.64$. Plasma central beta is less than $1 \%$. Toroidal rotation is found to reverse sign as the barrier is established. In contrast to many other such experiments ${ }^{14}$, this ITB occurs without reversed magnetic shear or high plasma rotation.

The ITB exhibits steep, spontaneous density peaking, a reduction in particle transport occurring without a central particle source. The ITB development occurs in the early phase of a dual frequency RF experiment, with central RF heating providing density control later in the discharge. For this ICRF EDA H-Mode, the minority resonance is at $r / a \sim 0.5$ on the high field side, beginning at $0.7 \mathrm{~s}$. EDA refers to enhanced $\mathrm{D}_{\square}$ radiation, which is high for high performance H-modes at high densities and temperatures. ITBs form in ohmic and ICRF heated plasmas, from fully equilibrated H-modes. In Fig. 2 are radial profiles of the plasma electron density, electron temperature and ion temperature for times within the L-mode, H-mode and early ITB phases. The times of interest for gyrokinetic simulations near the time of ITB onset are $0.8 \mathrm{~s}$ and $0.9 \mathrm{~s}$.

The experiment was analysed with the TRANSP $\operatorname{code}^{15}$, including a sawtooth model which causes the safety factor, $q$, to drop below unity by $\sim 10 \%$ at each sawtooth. The TRANSP results were processed with TRXPL ${ }^{16}$, averaging over $50 \mathrm{~ms}$ at the time of interest. As the sawtooth period is $10 \mathrm{~ms}$, a sawtooth-averaged condition is used for the simulations. The plasma had a normal shear profile, with q monotonic. Error in the experimental measurements of all data is estimated to be on the order of $10-20 \%$. The impurity and minority ions at $0.9 \mathrm{~s}$ are estimated to be $3 \%$ boron and $4 \%$ hydrogen. The walls of C-Mod are molybdenum, regularly 
coated with boron (from $\mathrm{D}_{2} \mathrm{~B}_{6}$ boronization) for edge density control. Examination of bolometer profiles indicates molybdenum levels $<0.1 \%$. The low $Z$ impurity level is estimated at $3 \%$ from visible bremsstrahlung measurements, yielding $Z_{\text {eff. }}$ The impurity ion is identified as boron, although the low $Z$ impurities may be actually $2 \%$ boron and $1 \%$ carbon. Boron and carbon are so similar in collisional properties that only boron impurity ions are included in the simulations. No oxygen is thought to have been present. The hydrogen to deuterium ratio, $4 \%$ at $0.9 \mathrm{~s}$, was obtained from spectroscopic measurements of $\mathrm{D}_{\square}$ and $\mathrm{H}_{\square}$ radiation.

Radio frequency heating of the hydrogen minority causes the hydrogen temperature $\left(T_{h}=2 E_{h} / 3 k\right)$ to be peaked around the half radius, with a less radially peaked hydrogen density profile. The ion distribution function is not thought to have a high energy RF tail, due to the high density and collisionality.

Electron temperature data for the TRANSP analysis was taken from Thomson scattering as the electron cyclotron emission (ECE) signal is cut off during the ITB because of rising high electron density. The density profile data was obtained from inverted visible bremstrahlung measurements, adjusted for the $Z_{\text {eff }}$ and temperature dependence. The plasma ion temperature profile in TRANSP was calculated on the basis of neutron data and the assumption that $\square_{\mathrm{i}}$ is proportional to the neoclassical ion diffusivity ${ }^{17}, \square_{i}^{\text {Chang-Hinton }}$. This leads to $T_{i}(r)$ being broader, and slightly lower than $T_{e}(r)$. While the high density of C-Mod suggests that $T_{i}=T_{e}$ would be a good assumption in the plasma core, the data are consistent with either ion thermal loss model.

\section{GYROKINETIC SIMULATIONS}

The transport of particles and energy in high temperature fusion plasmas is widely believed to result from the turbulence of drift wave fluctuations. In general, driving forces for the microturbulence arise from temperature and density gradients in $\square *$, mediated by the effects of 
passing ions and of trapped electrons. Stabilization of turbulence can be achieved through high values of magnetic shear $\hat{s}, q, \square \square$ impurities, collisional effects, and nonzero $T_{e}$. The competition among many driving and stabilizing forces requires computational methods to analyse a particular experimental situation.

Simulations of the C-Mod experiment examine stability before the ITB develops ( 0.8 and $0.9 \mathrm{~s}$ ) at three locations: the plasma core at $r / a \sim 0.25$, the region where the ITB forms at $r / a \sim 0.45$ and outside the ITB at $r / a \sim 0.65$. Calculations were carried out on the USDOE IBM RS/6000SP computers with 64 parallel processors distributed on 4 nodes, using approximately 5,000 hours of computation on the IBM SP.

The simulations solve the gyrokinetic Vlasov-Maxwell system and are run out for 10,000-50,000 time steps, until the microinstability growth rates, $\square$ and real frequencies, $\square$, are verified to have converged and the usual measure of the electrostatic potential, $\ln \mid \square^{2}$, is verified to be linearly increasing, in cases designated unstable. The fully electromagnetic calculations include the complete nonadiabatic electron response, and four plasma species: electrons, deuterium, boron impurity and fast hydrogen ions. They cover the full range of drift mode wavenumbers, including ion temperature gradient mode (ITG), trapped electron mode (TEM) and electron temperature gradient mode (ETG) wavelengths, $k_{\square} \square_{s}=0.1$ to 80 , where $\square_{s}=\left(m_{i} T_{e}\right)^{0.5} / e B$. Drift mode microturbulence is denoted ITG/TEM for $k_{\square} \square_{s}$ ranging from 0.1 to 1 , as these instabilities are often hybrid modes characterized by both ITG and TEM behavior. From $k_{\square} \square_{s}=2$ to 10 the instabilities are TEM, while above $k_{\square} \square_{s}=10$ the microturbulence is pure ETG, driven by passing electrons only.

Simulations were carried out for the ITB region early in the H-mode phase at $0.8 \mathrm{~s}$ and in fully equilibrated $\mathrm{H}$-mode at $0.9 \mathrm{~s}$, just before ITB onset. In general, ITBs develop from fully 
equilibrated H-modes. The spectra of weak, fully converged ITG/TEM modes at $0.8 \mathrm{~s}$ and $0.9 \mathrm{~s}$ are very similar (Fig. 3) as are the TEM and ETG mode spectra. At the earlier time most plasma parameters are the same within $10 \%$, except $-a \square n_{s} / n_{s}, \sim 0.07$ rather than 0.42 . All subsequent simulations in this paper concern plasma conditions at $0.9 \mathrm{~s}$ in fully equilibrated $\mathrm{H}$-mode.

In the plasma core, weakly unstable ITG/TEM modes, apparently well converged in time, with $0.3<k_{\square} \square_{s}<0.6(0.02 \mathrm{MHz})$ occur in simulations of five $2 \square$ field periods along the field line. These instabilities were driven by boundary conditions, having very low growth rates $(<0.005$ $\mathrm{MHz}$ ) when the field lines were extended to seventeen $2 \square$ field periods. They rotate in the electron diamagnetic direction with peaks in the eigenfunctions from particle trapping regions (Fig 4a). Convergence studies in field line length were necessary in the plasma core, but not at or outside the ITB region. $\square_{i}^{e}=\left(\square T_{i} / T_{i}\right) /\left(\square n_{e} / n_{e}\right)=0.94$ in the plasma core, so that the ITG/TEM instability is not expected there. $\square=0.94 \%$ in the plasma core, so that these modes are unlikely to be kinetic ballooning instabilities. These modes in the plasma core are probably spurious, arising from box size and were not pursued further. Figures $4 b$ and $4 c$ show the eigenfunctions of the ITG/TEM electrostatic potential at and outside the ITB region for the same wavenumber.

Figure 5 shows the growth rates and real frequencies at $0.9 \mathrm{~s}$ for radii at and outside the ITB region. In the barrier region $(r / a \sim 0.45)$ are weak ITG/TEM modes for $0.1<k_{\square} \square_{s}<0.4$, while outside the ITB region ITG/TEM drift modes are strongly unstable at $0.1<k_{\square} \square_{s}<0.8$. The TEM (usually found near $k_{\square} \square_{s} \sim 1$ ) is stable everywhere. The ETG is strongly unstable at and outside the barrier, with maximum growth rates near $k_{\square} \square_{s} \sim 20$. Previous gyrokinetic simulations ${ }^{9,10}$ of this plasma were based on ratios of $\square_{d-i} / \bigsqcup_{k-i}$ which were too high by factors of $(36,20,6)$ for plasma radii $(0.25,0.45,0.65)$. Such high ion collisionalities completely stabilized the weak 
ITG/TEM mode in the barrier region and led to apparent quiescent microturbulence in nonlinear simulations there, but the $\square_{d-i} \square_{k-i}$ values have been corrected in the present work.

At ITB onset the fastest growing ITG/TEM mode growth rates $(0 . \mathrm{MHz}, 0.026 \mathrm{MHz}$, $0.13 \mathrm{MHz})$ increased with increasing radii, $r / a=(0.25,0.45,0.65)$. Figure 6 shows the most important dimensionless driving forces, which usually destabilize or stabilize ITG/TEM instabilities, at these values of $r / a$. The $\square^{e},-a \square T_{e} / T_{e},\left[a \square n_{e} / n_{e}\right]^{-1}$ and $\hat{s}$ parameters increase with radius, while $Z_{\text {eff }}$ is assumed constant and $T_{i} / T_{e}$ is nearly constant. In the next section the barrier region $\square_{i}^{f}$ is shown to be less effective than the critical temperature gradient in destabilizing ITG. Effects of scaling the normalized temperature and density gradients on ITG/TEM instability were studied for the plasma conditions in the barrier region. In these cases, all species were scaled by the same multiplicative factor, and all other plasma conditions were held constant (Figs. 7-9). We defer investigation of the role of magnetic shear on ITG stability in the barrier region until measurements are available.

\section{DISCUSSION}

\section{A. Comparison with theoretical and simulation models}

At the onset time for ITB formation, the barrier region has a sufficiently flat density profile that the toroidal ITG mode threshold is a critical temperature gradient ${ }^{18}$, rather than a critical $\square_{i}^{f}$. The usual value $\square_{i}^{\text {crit }}=1.2$ is not valid at ITB onset time to estimate ITG instability. If $\square_{h} \equiv L_{n} / R>\square_{\imath}^{\text {crit }}=0.9 /\left[1+1 /\left(T_{e} / T_{i}\right)\right][1+2 \hat{s} / q]$, the critical parameter $\square_{i}^{\text {crit }}$ becomes

$$
\square_{i}^{\text {crit }}=(4 / 3)\left[1+1 /\left(T_{e} / T_{i}\right)\right]\left[L_{n} / R\right][1+2 \hat{s} / q] .
$$

Here $L_{n}$ is the electron density scale length. At the time of onset at radii $(r / a=0.25,0.45,0.65), \square$ has the values $(0.5,0.8,8.3)$, higher than the critical thresholds $\square_{\imath}^{\text {crit }},(0.22,0.18,0.17)$. Thus $\square_{i}^{\text {crit }}$ 
is higher than 1.2 , namely $(3.2,5.6,56)$ at these radii. The experimental values of $\square_{i},(0.94,6.5$, 68) are well below criticality in the core and above threshold in the ITB region and outside the ITB, in agreement with the linear gyrokinetic simulations. At $0.8 \mathrm{~s}$ the analytic model predicts that in the ITB region $\square_{i}^{\text {crit }}=35$, equal to the experimental value. At this time the model predicts that the plasma is at marginal stability, although the gyrokinetic simulations show that drift mode stability is virtually unchanged between 0.8 and $0.9 \mathrm{~s}$.

Garbet ${ }^{19}$ points out that for most radii plasmas are not usually marginally stable, but only when there are conditions of high heat and particle transport. This is the case at ITB onset in the barrier region. Here the C-Mod plasma is above marginal stability, well above the critical value of the normalized temperature gradient $R[\square T / T]_{c r i t}=0.8 R[\square T / T]_{\text {exp }}=6.9$, and exhibits weak ITG/TEM instability (Fig. 7a). The maximum growth rate increases linearly with $\square \square / \square /[\square \square / \square] \exp$ up to a multiplicative factor of 2, applied to all four species. Above $(\square T / T) /[\square T / T]_{e x p}=4$, a different root at long wavelength $\left(k_{\square} \square_{s}=0.1\right)$ becomes the most unstable mode (Fig. 7b) and the maximum growth rates rise by an order of magnitude with broadened, nonparabolic spectra. For $(\square T / T) /[\square T / T]_{\exp } \square 2$ the growth rate spectrum $\bigsqcup^{\mathrm{TG}}\left(k_{\square} \square\right.$ s $)$ has a parabolic shape, peaked at $0.2-0.3 k_{\square} \square_{s}$ (Fig. 7c). Just above the experimental normalized temperature gradient, the growth rate shows nonmonotonic behavior, decreasing slightly, when the wavenumber of the fastest growing mode switches between $k_{\square} \square_{s}=0.2$ and 0.3 (Fig. 7c). At $(\square T / T) /[\square T / T]_{\text {exp }}=2, \square\left(k_{\square} \square_{s}\right)$ is as high as that predicted for strong ITG/TEM turbulence outside the ITB region, at $r / a=0.65$.

From extensive linear GS2 simulations, Jenko ${ }^{20}$ has formulated an algebraic ETG critical temperature gradient, which reduces to two previous analytic theories ${ }^{21,22}$ for ITG modes in appropriate limits. The ETG critical temperature gradient formulation can be extended to ITG 
modes if $\square_{i}$ replaces $\square_{e}, L_{T i}$ replaces $L_{T e}$, and $T_{i} / T_{e}$ replaces $T_{e} / T_{i}$, as geometrical effects are likely to be the same for electrons and ions. We do not address $Z_{\text {eff }}$ dependence, as the role of $Z_{\text {eff }}$ is complex in controlling the stability of ITG modes ${ }^{23}$. This leads to

$$
\left(R / L_{T i}\right)_{\mathrm{crit}}=\max \left\{\left(1+\square(1.33+1.91 \hat{s} / q)\left(1-1.5 \square[1+0.3 \square d \square / d \square], 0.8 R / L_{n}\right\},\right.\right.
$$

In the barrier region $\hat{s}=0.96, q=1.32, \square=1.16, \square=1.24, \square \equiv r / R_{o}=0.14,(\square / \square)(d \square / d \square=0.08$.

The critical ion temperature gradient from our linear simulations of the barrier region at ITB onset, $\left(R / L_{T i}\right)_{\text {crit }}=6.9$, is higher than predicted by the above formula, 4.9. (Note that the ion temperature scale length here is normalized by $R=0.69$, to simplify comparison with Ref. 20 . In the rest of the paper gradient normalizations use $a=0.22$ ). Our simulations were based on numerical equilibria and include geometrical effects as in the standard ETG model ${ }^{20}$, but also include trapped particle effects, electron-ion collisions and impurity ions, not included there. This difference in critical temperature gradients suggests that the transport barrier is more easily formed near $r / a=0.45$ than for typical high turbulence plasma regions which were the basis of the critical temperature gradient models. It also appears that including trapped particle effects, electron-ion collisions and impurity ions is important in modeling ITB formation. This physics was missing from earlier gyrokinetic simulations and from the analytic derivations used to develop the standard critical gradient models.

Dimits $^{24}$ showed that collisionless, electrostatic, nonlinear ITG simulations lead to an upshift in the critical temperature gradient of about $20 \%$, similar to the difference between the difference between our simulated critical temperature gradients and the experimental temperature gradient. The C-Mod ITB region has $q \sim 1.3$, as for the largest Dimits upshifts and C-Mod turbulence is expected to be electrostatic. Although only weak ITG modes are linearly 
destabilized, nonlinear ITG simulations may show the ITB region is at marginal stability at the onset time.

The ITG instability is robust at all values of the logarithmic density gradient multiplier, (Fig. 8) changing from ITG to TEM with increasing scaling factors, as expected from the ITG/TEM stability diagram ${ }^{19}$. The maximum growth rate increases linearly with $\square n_{s} / n_{s}$ up to multiplicative factor 4 , becoming increasingly sensitive to the multiplicative factor above 6 . The real frequency of the fastest growing mode decreases slowly up to scaling factor 4 and then at 6 the solution transitions from an ITG-like to a TEM-like root (Fig. 9). Kinetic theory has shown in general that density peaking may stabilize or destabilize the ITG/TEM mode, depending on plasma collisionality, trapped electron fractions and $T_{i} / T_{e}^{25}$. At ITB onset in the barrier region, density peaking further destabilizes the ITG/TEM mode in the C-Mod case. For the C-Mod ITB onset time, we find the growth rates in the barrier region are much more sensitive to increased $-a \square T_{s} / T_{s}$ than to $-a \square n_{s} / n_{s}$.

\section{B. Comparison with transport analysis of experiment}

In principle, predictions for experimentally measured heat and particle fluxes and transport coefficients can be found from nonlinear gyrokinetic simulations for each species. However, because C-Mod has such high plasma density, error estimates for heat fluxes and diffusivities can be as large as $\pm 70 \%$, so that

$$
\square_{e f f}=\left(\square_{i} n_{i} \square T_{i}+\square_{e} n_{e} \square T_{e}\right) /\left(n_{i} \square T_{i}+n_{e} \square T_{e}\right)
$$

is reported, rather than $\square_{e}$ and $\square_{i}$. In Fig. 10 is shown the experimental $\square_{\text {eff }}$ and the neoclassical ion thermal conductivity ${ }^{17}, \square_{i}^{\text {Chang-Hinton }}$ at the ITB onset time. The large differences between the experimental effective heat diffusivity and $\square_{i}^{\text {Chang-Hinton }}$, both at and outside the ITB region, indicate that microturbulent based anomalous transport is likely to be important at both locations. 
A good quantitative comparison of simulation against experiment is possible in the plasma core, since there the toroidal velocity and velocity shear are zero. No anomalous transport is found in the core (Fig. 10), consistent with simulations which show no strongly unstable linear microinstabilities there. It is unlikely that nonlinear gyrokinetic calculations will yield significant ITG/TEM microturbulence in the core, given the flat density and temperature profiles. Subcritical turbulence has only been identified in gyrofluid calculations near the plasma edge ${ }^{26}$. Self-sustained core driftwave turbulence has not been observed to date at the low collisionality,

$$
C=0.51 \frac{\square_{e}}{c_{s} / L_{\square}} \frac{m_{e}}{M_{i}} \square q R \square_{L_{\square}}^{\square} \sim 10^{\square 3}<<1 .
$$

Extensive local nonlinear ITG/TEM simulations do not seem necessary for the plasma core.

Nonlinear ITG/TEM and ETG simulations at and outside the ITB region may underlie anomalous transport identified for this experiment at the ITB onset. Fully converged nonlinear simulations of both ITG/TEM and ETG modes would be needed for gyrokinetic benchmarking with experiment at and outside the ITB region, since it is not known how this is divided between the electron and ion channels.

In simulations of other tokamak experiments, both gyrofluid and gyrokinetic, ExB corrections are seen to be critical for satisfactory comparison with experiment ${ }^{27-33}$. Thermal diffusivities from nonlinear ITG/TEM simulations may be modified with the Waltz prescription ${ }^{27}$ for $\boldsymbol{E} \boldsymbol{x} \boldsymbol{B}$ shear corrections, making use of the linear growth rates. The approximate Waltz quench rule for reduction of nonlinear ITG/TEM drift-wave diffusion by $\boldsymbol{E} \boldsymbol{x} \boldsymbol{B}$ shear compares the maximum linear growth rate and the toroidal velocity shear, leading to a transport reduction factor $K . \square=K \square_{G S 2}=\square_{G S 2}\left[1-\operatorname{Min}\left\{1, G\left|\square_{x B} / \square_{I T G}\right|\right\}\right]$, where $0.3<G<3.0$ and $\left[E_{x B}=R\right.$ $\left(B_{\square} B\right) d / d r\left[V_{t o r} /\left(R_{o}+r \cos \square\right]\right.$. This particular form of $\left[E_{x B}\right.$, which ignores contributions from $v_{p o l}$ 
and $\square P$ is a limiting case of the more general $\boldsymbol{E} \boldsymbol{X} \boldsymbol{B}$ shearing rate ${ }^{29}$. We note that if the contributions from $v_{p o l}$ and $\square P$ (measurements are not available) were included in the analysis, quantitative changes in the results could occur. A more accurate treatment is possible with nonlinear simulation which incorporates toroidal velocity shear along with evolving zonal flows, for instance, with the GYRO flux-tube code ${ }^{34}$.

In the experiment simulated, the toroidal velocity at ITB onset is zero in the single point measurement near the plasma center. Radial profile measurements of toroidal velocity on similar C-Mod ITB experiments ${ }^{13}$ have been reported during pre-ITB plasmas, but are not available for the ITB experiment simulated. Fig. 11 shows velocity measurements ${ }^{13}$ representative of C-Mod ITB plasma conditions, averaging three ITB cases. It is important to point out that toroidal velocity evolution can vary among ITB cases, for example in a recent higher power ITB experiment ${ }^{8}$ the toroidal velocity does not reverse sign, in contrast to the experiment simulated here. The $v(r)$ data ${ }^{13}$ do not show the central velocity decreasing through zero during the H-mode to ITB phase, as occurs for the experiment simulated (Fig. 1). When $v_{t o r}(0)$ of Ref. 13, at $1.05 \mathrm{~s}$, the central plasma pressure is $0.19 \mathrm{MPa}$ and the plasma has a fully developed ITB.

In general, ITBs form from fully equilibrated, EDA H-mode plasmas, which exhibit flat velocity profiles ${ }^{13}$. In Figure 11 are extrapolations of the measured core velocity for the simulated shot. A flat profile with $v_{t o r}(0)=0$ is denoted with open squares. Maximum velocity shearing rates are estimated from the blue squares, representing typical velocity error bars of $\pm 0.1 \times 10^{4} \mathrm{~m} / \mathrm{s}$ within and at the ITB region and $\pm 0.2 \times 10^{4} \mathrm{~m} / \mathrm{s}$ outside the ITB. In Table I are compared the maximum linear growth rates of ITG instabilities, the $\boldsymbol{E} \boldsymbol{X \boldsymbol { B }}$ shearing rates and the Waltz quench factors $K$ for the extrapolated data points (open and solid squares), based on 
equilibrated H-mode plasmas as discussed above. $\boldsymbol{E} \boldsymbol{X} \boldsymbol{B}$ shear suppression is subdominant except possibly in the ITB region.

At the onset time $(0.9 \mathrm{~s})$ of the shot simulated, $v(0)$ is known to be zero. Nonlinear gyrokinetic simulations could be quantitatively benchmarked against transport analysis for this experiment outside the ITB region, without the inaccuracies inherent in calculations which do not include zonal and $\boldsymbol{E} \boldsymbol{x \boldsymbol { B }}$ flow selfconsistently. $\boldsymbol{E} \times \boldsymbol{B}$ shear suppression may be important in benchmarking the gyrokinetic model against experiment in the ITB region, because of weakly unstable ITG/TEM turbulence. While linear simulations provide a good model benchmark in the plasma core, nonlinear ITG/TEM and ETG simulations are still essential for experimental validation of the gyrokinetic drift wave model at and outside the ITB. Considering the impact of $V(r)$ on gyrokinetic model validation and the limited resources available, fully converged, nonlinear ITG gyrokinetic simulations (which would presently require more than 100,000 hours of computation) should be limited to experiments having in situ velocity profile measurements.

\section{Conclusion}

Fully electromagnetic, linear, gyrokinetic simulations of the Alcator C-Mod ITB during off-axis RF heating, including the complete electron response, and following four plasma species, show ITG/TEM microturbulence is suppressed in the plasma core before barrier formation, without recourse to the usual requirements of velocity shear or reversed magnetic shear. Strong ITG/TEM and ETG drift wave turbulence are identified outside the barrier region. Linear microstability analysis is qualitatively consistent with the experimental transport analysis, showing low transport inside and high transport outside the ITB region before barrier formation.

Since there are no strong drift wave instabilities at the ITB region, microturbulent driving forces are not strong enough to provide outward anomalous particle transport across the barrier 
region even before ITB formation. The good confinement appears to result from a combination of factors in the ITB region $(q, \hat{s},-\square n / n,-\square T / T)$, which are not sufficient to stabilize turbulent microstabilities outside the plasma core. Strong dependence of the barrier region ITG instability on the normalized temperature gradient suggests that local RF heating causes the ITB in this experiment. In the barrier region the experiment is above marginal stability, and the critical temperature gradient in the barrier region is higher than expected from standard models. For ITB predictions and reactor considerations these models should be extended to include impurities, nonadiabatic electrons and collisionalities. Integrating velocity profile data, transport analysis and linear growth rates provides a qualitative understanding of the microscopic physics underlying turbulent transport at the ITB onset time on C-Mod and a good, quantitative gyrokinetic model benchmark in the plasma core.

\section{ACKNOWLEDGEMENT}

We are glad to acknowledge the experimental and diagnostic teams at Alcator C-Mod. J. Baumgaertel, a student at University of Washington, Seattle, held a Science Undergraduate Laboratory Internship at the Princeton Plasma Physics Laboratory during the summer of 2004. Contributions by D. Ernst, MIT, to the early stages of this work are gratefully acknowledged. We especially wish to acknowledge stimulating discussions about the experiment with S. Wolfe, S. Wukitch, B. Lipschultz, and J. Terry at MIT and about microinstability physics and gyrokinetic modeling with E. Belli, R. Budny, and W. Lee of Princeton Plasma Physics Laboratory, Princeton University, with C. Bourdelle, Euratom-CEA, France and with B. Scott and F. Jenko, Max-Planck-Institut fur Plasmaphysik, EURATOM, Garching, Germany. We also thank G. Schilling, PPPL, and E. Marmar and M. Porkolab, MIT, for their support. Research was carried out under U. S. DOE Contract DE-AC02-76CH03073. 


\section{References}

[1] I. H. Hutchinson, R. L. Boivin, F. Bombarda et al., Phys. Plas. 1, 1511 (1994).

[2] “ITER Physics Basis”, Nuclear Fusion, 39, 2137-2638 (1999).

[3] M. Kotschenreuther, G. Rewoldt and W. M. Tang, Comp. Phys. Comm. 88, 128 (1995).

[4] W. Dorland, F. Jenko, M. Kotschenreuther and B. N. Rogers, Phys. Rev. Lett. 85, 5579 (2000).

[5] C. L. Fiore, J. E. Rice, P. T. Bonoli, et al., Phys. Plas. 8, 2023 (2001).

[6] S. Wukitch, R. L. Boivin, P. T. Bonoli, et al., Phys. Plas. 9, 2149 (2002).

[7] J. E. Rice, P. T. Bonoli, E. Marmar, et al., Nuclear Fusion 42, 510 (2002).

[8] C. L. Fiore, P. T. Bonoli, D. R. Ernst, et al., "Internal Transport Barrier Production and Control in Alcator C-Mod", to appear in Proceedings of the $31^{\text {th }}$ EPS Conference on Plasma Physics and Controlled Fusion, London, England (2004), and Plasma Physics and Controlled Fusion (in press).

[9] M. H. Redi, C. Fiore, P. Bonoli, et al., Proceedings of the $29^{\text {th }}$ EPS Conference on Plasma Physics and Controlled Fusion, Montreux, Switzerland, 2002, edited by R. Behm, C. Varandas (European Physical Society, Lausanne, 2002) Vol. 26B, Paper 1.082.

[10] M. H. Redi, R. Bell, P. Bonoli et al., Proceedings of the $30^{\text {th }}$ EPS Conference on Plasma Physics and Controlled Fusion, St. Petersburg, Russia, 2003, edited by R. Koch, S. Lebedev (European Physical Society, Lausanne, 2003), Vol. 27A, Paper P4.94.

[11] M. H. Redi, C. L. Fiore, W. Dorland, et al., "Benchmarking Nonlinear Turbulence Simulations on Alcator C-Mod", to appear in Proceedings of the $31^{\text {th }}$ EPS Conference on Plasma Physics and Controlled Fusion, London, England (2004), Paper P2-163.

[12] D. R. Ernst, P. T. Bonoli, P. J. Catto, et al., Phys. Plas. 11, 2637 (2004). 
[13] J. E. Rice, P. T. Bonoli, C. L. Fiore, et al. Nuclear Fusion 43, 781 (2003).

[14] R. C. Wolf, Plasma Physics and Controlled Fusion 45, R1-R91 (2003).

[15] R. J. Hawryluk, in Physics of Plasmas Close to Thermonuclear Conditions, edited by B.

Coppi, G. G. Leotta, D. Pfirsch, R. Pozzoli, and E. Sindoni (Pergamon, Oxford, 1980), Vol. 1, p. 19.

[16] D. McCune, G. W. Hammett, C. Bourdelle, private communication (2002).

[17] C. S. Chang, F. L. Hinton, Phys. Fluids 25, 1493 (1982).

[18] J. A. Wesson, Tokamaks, Oxford University Press, New York, NY (1997) p. 403.

[19] X. Garbet, P. Mantica, C. Angioni, et al., "Physics of Transport in Tokamaks", to appear in Proceedings of the $31^{\text {th }}$ EPS Conference on Plasma Physics and Controlled Fusion, London, England (2004), and Plasma Physics and Controlled Fusion (in press).

[20] F. Jenko, W. Dorland, G. W. Hammett, Phys. Plas. 8, 4096 (2001).

[21] F. Romanelli, Phys. Fluids B1 1018 (1989)

[22] T. S. Hahm, W. M. Tang, Phys. Fluids B1, 1185 (1989).

[23] M. Kotschenreuther, W. Dorland, M. Beer, G. W. Hammett, Phys. Plas. 2, 2381 (1995).

[24] A. M. Dimits, G. Bateman, M. A. Beer et al., Phys. Plasmas 7, 969 (2000).

[25]F. Romanelli and S. Briguglio, Phys. Fluids B2, 754 (1990).

[26] B. D. Scott, Plasma Phys. Control. Fusion 45, A385-A398 (2003).

[27] R. E. Waltz, R. L. Dewar, X. Garbet Phys. Plas. 5, 1784 (1998).

[28] D. W. Ross, R. V. Bravenec, W. Dorland, et al. Phys. Plas. 9, 177-184 (2002).

[29] T. S. Hahm, K. H. Burrell, Phys. Plas. 2, 1648 (1995).

[30] R. E. Waltz, G. D. Kerbel, J. Milovich, G. W. Hammett Phys. Plas. 2, 2408 (1995). 
[31] R. V. Budny, R. Andre, A. Becoulet, et al. Plasma Physics and Controlled Fusion 44, 12751228 (2002).

[32] D. W. Ross, W. Dorland, Phys. Plas. 9, 5031-5035 (2002).

[33] R. V. Budny, R. Andre, C. D. Challis, et al. Proceedings of the $30^{\text {th }}$ EPS Conference on Plasma Physics and Controlled Fusion, St. Petersburg, Russia, 2002, edited by R. Koch, S. Lebedev (European Physical Society, Lausanne, 2002), Vol. 27A, Paper O-3.4A(P3.88).

[34] J. Candy and R. E. Waltz, Phys. Rev. Lett. 91, 045001-1 (2003). 
Table I. Maximum linear growth rates of ITG instabilities, the $\boldsymbol{E} \boldsymbol{x} \boldsymbol{B}$ shearing rates and the Waltz quench factors $K(G=1)$ for the extrapolated toroidal velocity profiles designated by open and solid squares $(*)$ for three plasma regions.

\begin{tabular}{clllll}
\hline \hline$r / a$ & $\square^{n}{ }_{\text {ITG }}$ & $\square_{\times B}$ & $\square_{\times B}{ }^{*}$ & $K$ & $K^{*}$ \\
\hline 0.25 & 0 & 0 & $0.6 \times 10^{4} / \mathrm{s}$ & 1 & 1 \\
0.45 & $2.6 \times 10^{4} / \mathrm{s}$ & 0 & $1.4 \times 10^{4} / \mathrm{s}$ & 1 & 0.5 \\
0.65 & $13 \times 10^{4} / \mathrm{s}$ & 0 & $1.5 \times 10^{4} / \mathrm{s}$ & 1 & 0.9 \\
\hline \hline
\end{tabular}




\section{Figure Captions}

Figure 1. C-Mod ITB discharge with off-axis ICRF heating throughout the discharge and central ICRF heating applied after ITB established. Summary traces are ICRF power, stored plasma energy, line-averaged density, neutron rate, central ion temperature and toroidal rotation. Figure reproduced from Ref. 6, used with permission of the author.

Figure 2. a) Radial profiles of electron density showing evolution from L-mode $(0.7 \mathrm{~s})$ to $\mathrm{H}$ mode $(0.8,0.9 \mathrm{~s})$ and the ITB development phase, with peaked density profiles. The times of interest are 0.8 and $0.9 \mathrm{~s}$, before the ITB is established. The three radial locations, $r / a \sim 0.25,0.45$ and 0.65 , for the microstability calculations are shown.

b) Electron temperature profiles at times from 0.7 to $1.2 \mathrm{~s}$.

c) Ion temperature profiles at times from 0.7 to $1.2 \mathrm{~s}$.

Figure 3. The growth rates (top) and real frequencies (bottom) of the ITG modes in the ITB region at $0.8 \mathrm{~s}$ and $0.9 \mathrm{~s}$ are nearly identical, although the densities differ.

Figure 4. Real and imaginary parts of the electrostatic eigenfunctions of the $k_{\square} \square_{s}=0.2$ drift wave modes for a) the plasma core where $\left.\bigsqcup^{T T G}{ }_{\max } \square 0.005 \mathrm{MHz}, \mathrm{b}\right)$ the ITB region where $\bigsqcup^{T T G}{ }_{\max }=0.026$ $\mathrm{MHz}$ and c) outside the ITB region where $\bigsqcup^{T G}{ }_{\max }=0.13 \mathrm{MHz}$.

Figure 5.

a) Linear growth rates at and outside the ITB for $k_{\square} \square_{s}$ from 0.1 to 80 , the ITG/TEM, TEM and ETG range of wavenumbers. Frequency normalizations are $0.88 \mathrm{MHz}$ and $0.67 \mathrm{MHz}$ at $r / a=0.45$ and 0.65 .

b) Growth rates in the ITG range are shown on a linear wavenumber scale. At the time of onset, there are no unstable modes in the plasma core, weak instabilities at the barrier region and strong ITG instabilities outside the barrier region. 
c) Real frequencies at and outside the barrier region of ITG, TEM, ETG drift modes for $k_{\square} \square_{s}$ from 0.1 to 80 .

Figure 6. The normalized driving forces, which are important in stabilizing or destabilizing long wavelength drift mode microturbulence, are shown at ITB onset for three plasma radii.

Figure 7. (a) Maximum ITG growth rates in the barrier region increase with scaling factors $(\square T / T) /[\square T / T]_{\exp }$ up to 2 . The experiment is above marginal stability.

(b) Maximum ITG growth rates increase with scaling factors $(\square T / T) /[\square T / T]_{\exp }$ up to 8 . A new root is destabilized above scaling factor 4 .

c) The growth rate spectra $\square_{T G}\left(k_{\square} \square_{s}\right)$ show little TEM mode instability for scaling factors $(\square T / T) /[\square T / T]_{\exp }$ up to 2 .

Figure 8. Changes in barrier region growth rates (top) and real frequencies (bottom) of the fastest growing ITG modes, with scaling factors $(\square N / N) /[\square N / N]_{\exp }$ up to 10 . Growth rates show weaker dependence on scaling factor than for temperature scaling. There is no critical density gradient. The fastest growing mode changes from ITG-like to TEM-like near scaling factor 6.

Figure 9. Comparison of spectra of ITB region growth rates $\square_{T G}\left(k_{\square} \square_{s}\right)$ (top) and real frequencies $\square_{I T G}\left(k_{\square} \square_{s}\right)$ (bottom) for multiplicative factors $(\square N / N) /[\square N / N]_{\exp }$ up to 10 . TEM mode instabilities appear at scaling factors greater than 5-6.

Figure 10. Radial profiles of $\square_{\text {eff }}$ and the Chang-Hinton neoclassical ion conductivity from transport analysis of the experiment at $0.9 \mathrm{~s}$.

Figure 11 . Toroidal velocity data ${ }^{13}$ and extrapolated velocities (open squares) based on measured velocity of zero, assuming a flat, equilibrated EDA H-mode $v(r)$ profile. Extrapolated velocity profile based on typical velocity error bars is shown with solid squares. 


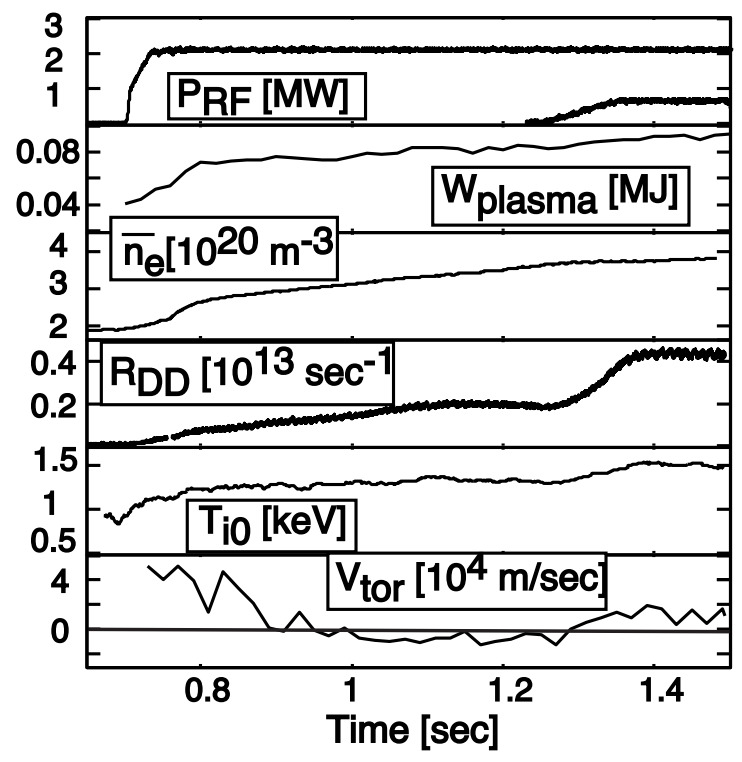

Fig. 1

Redi 

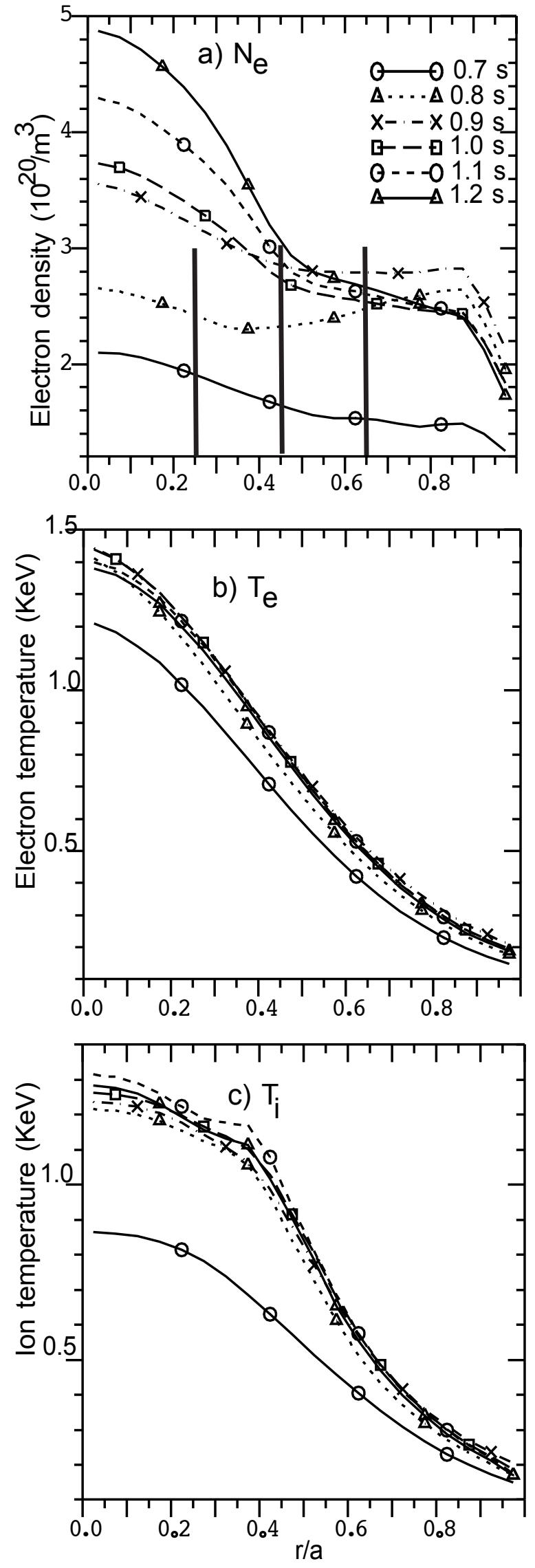

Fig. 2, Redi 

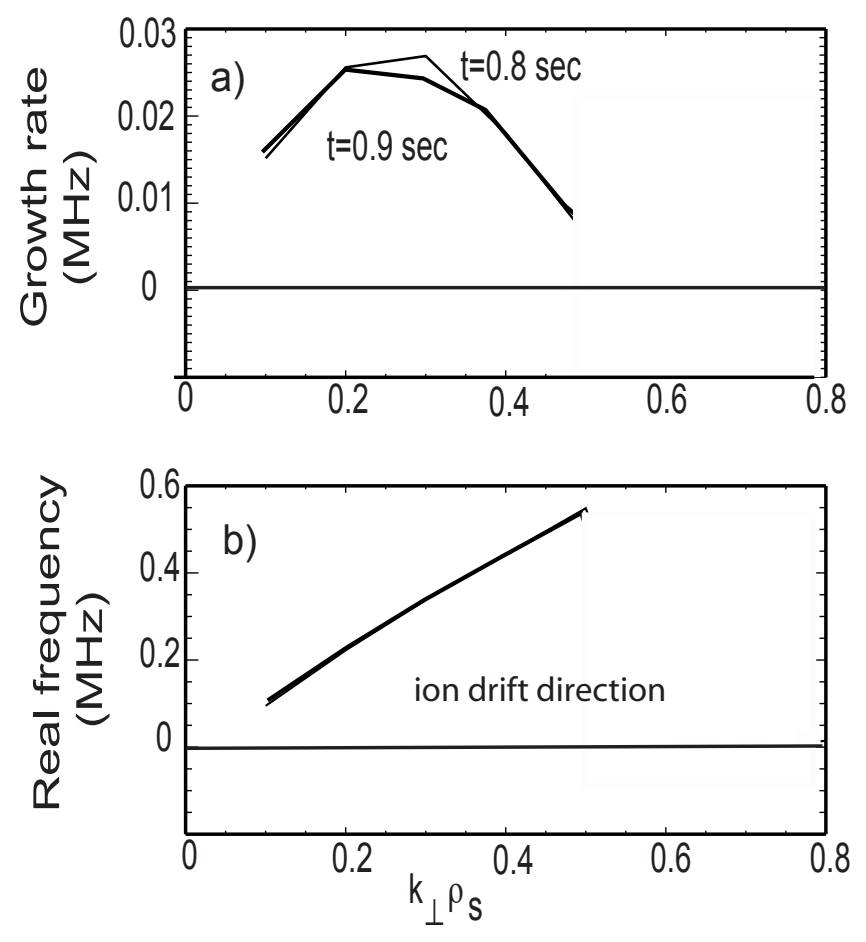

Fig 3

Redi 

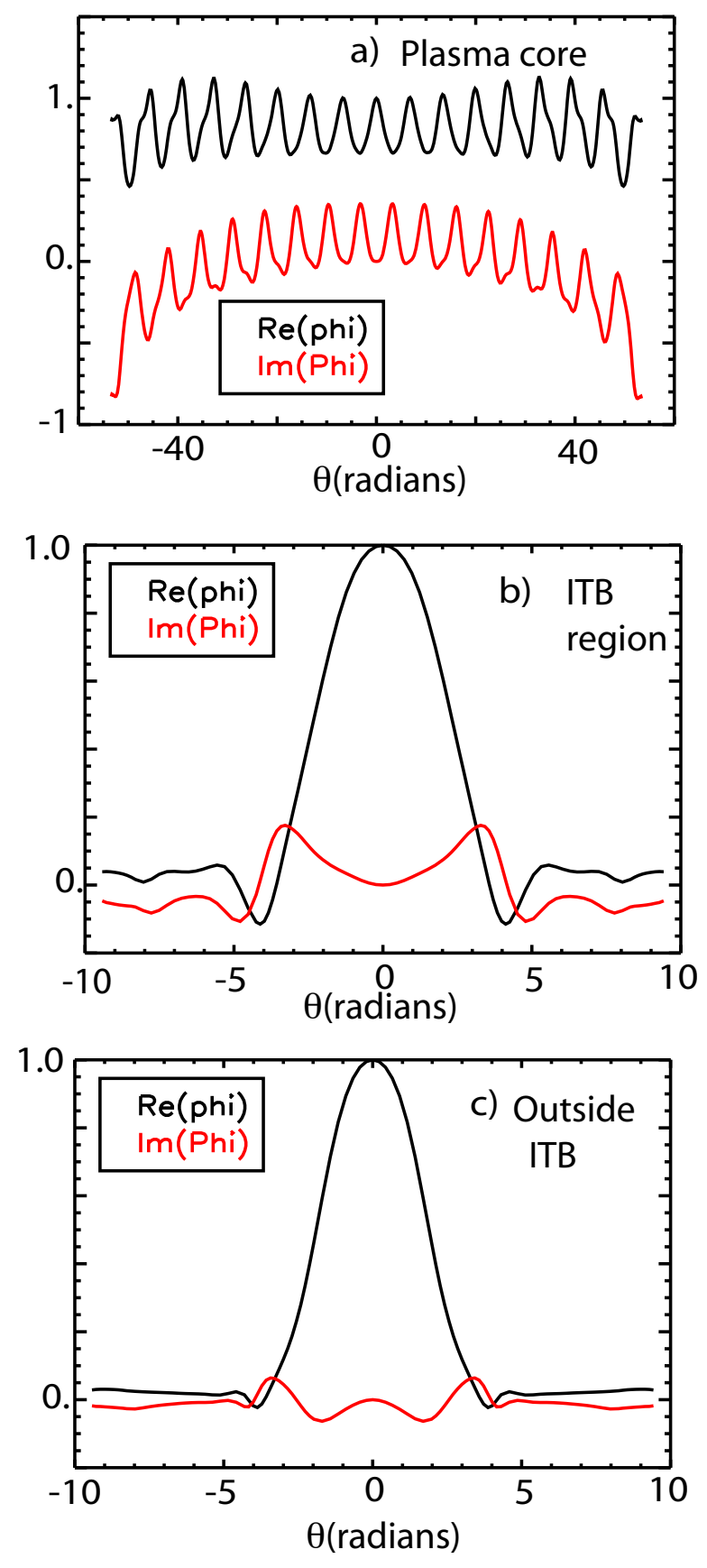

Fig. 4

Redi 

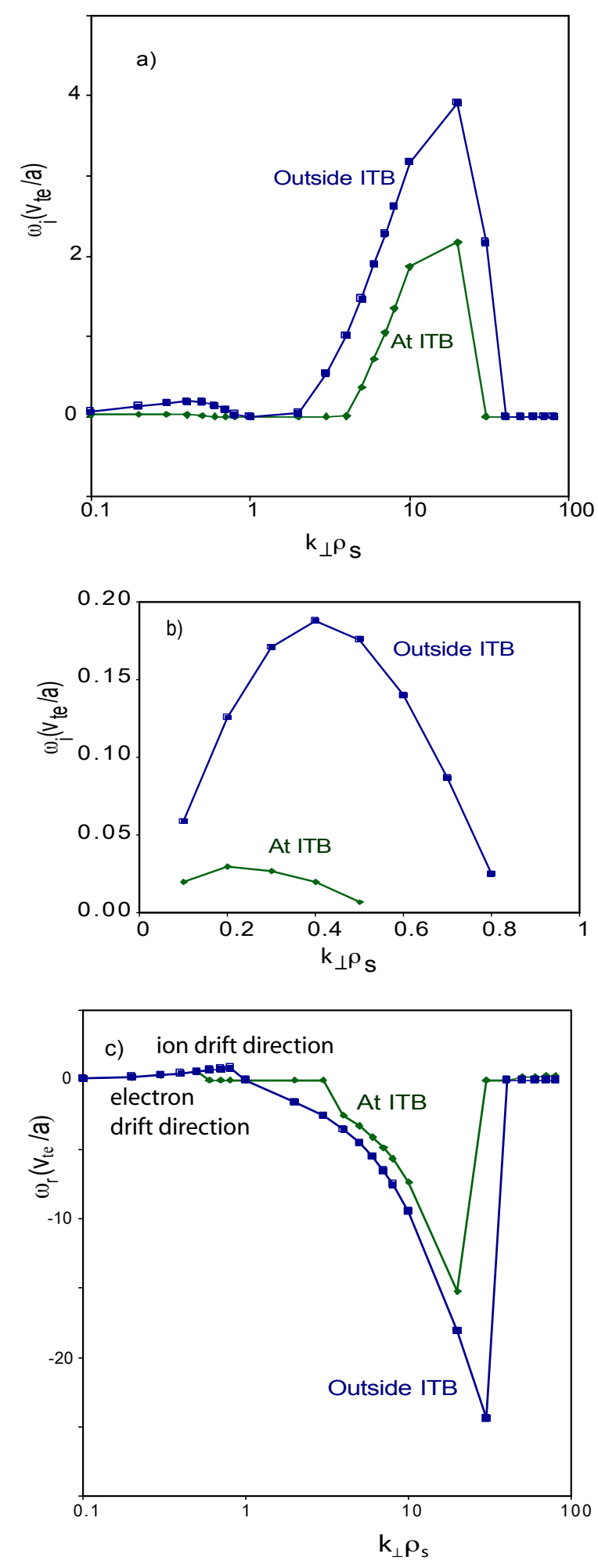

Fig 5

Redi 


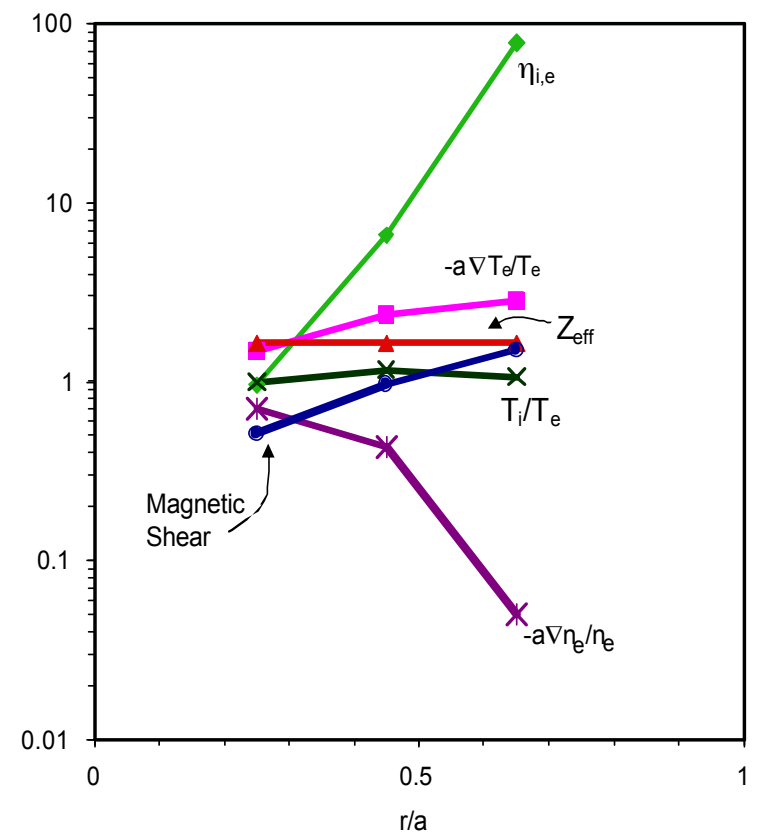

Fig. 6

Redi 

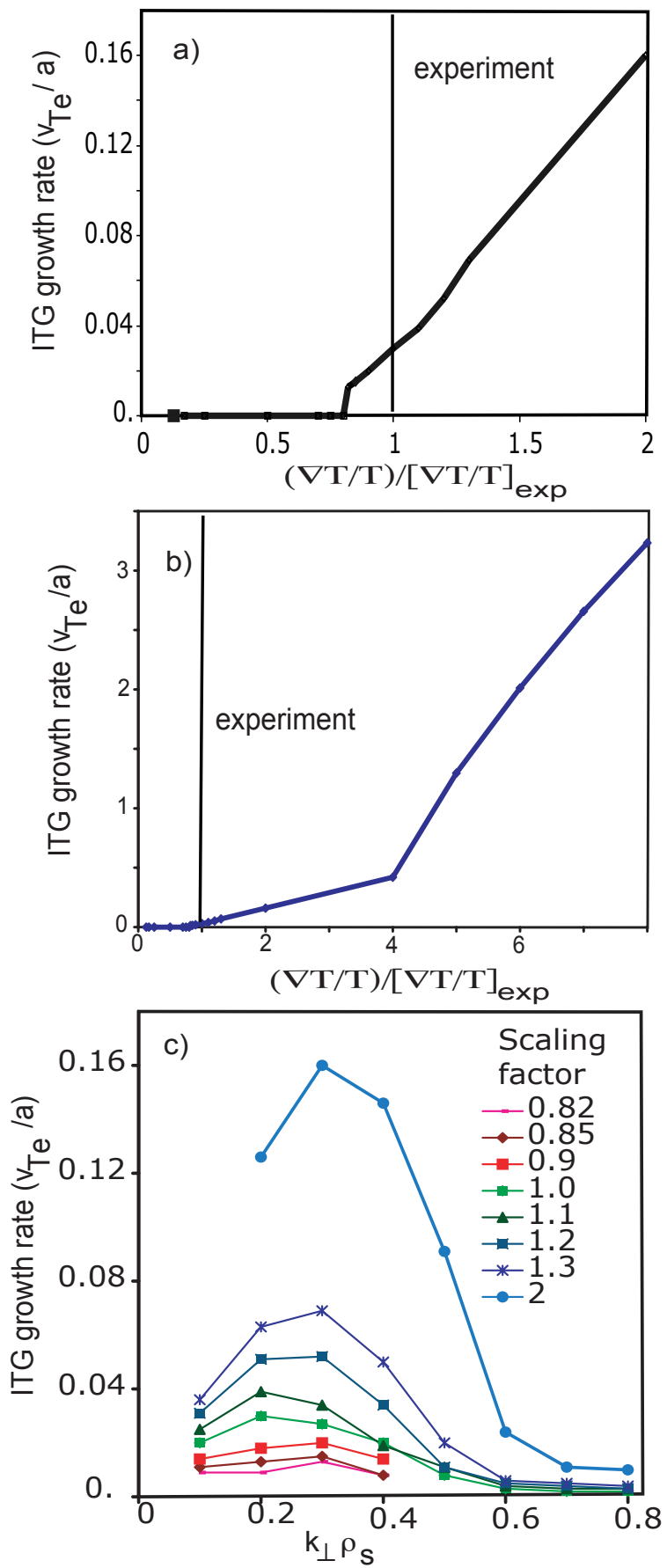

Fig 7 

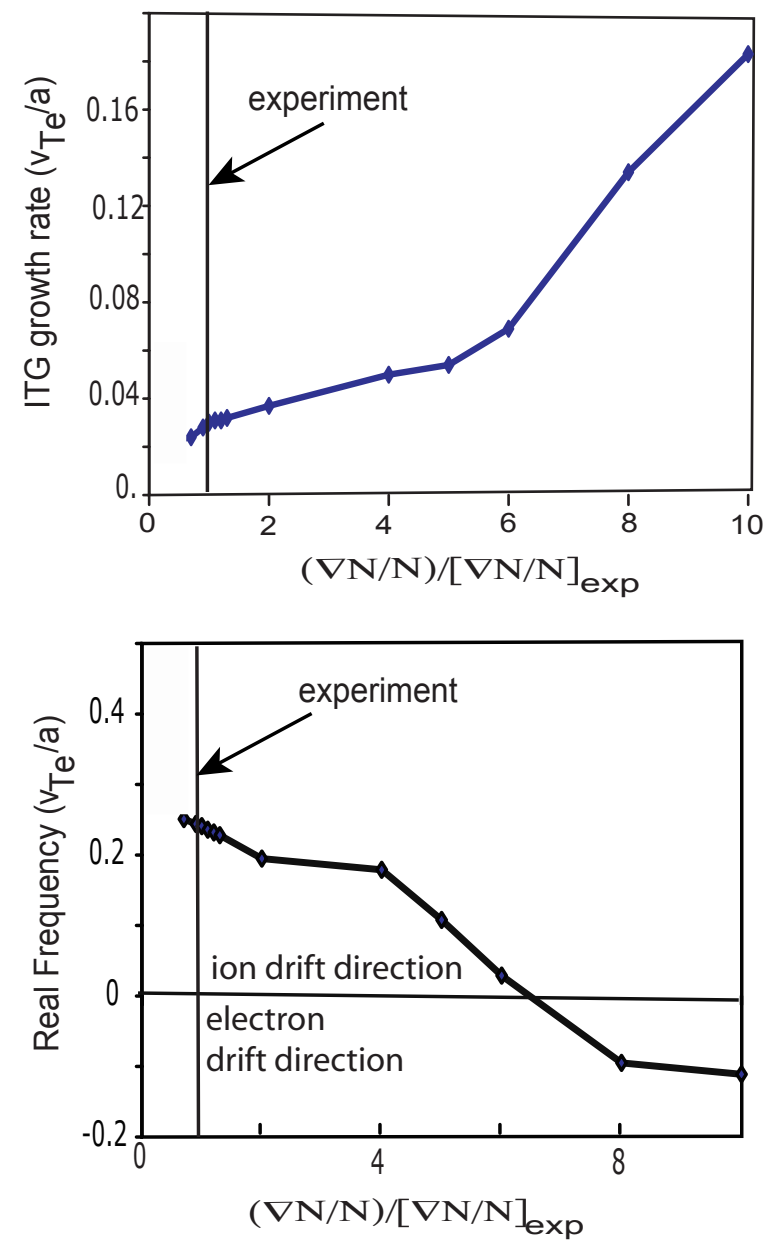

Fig. 8 


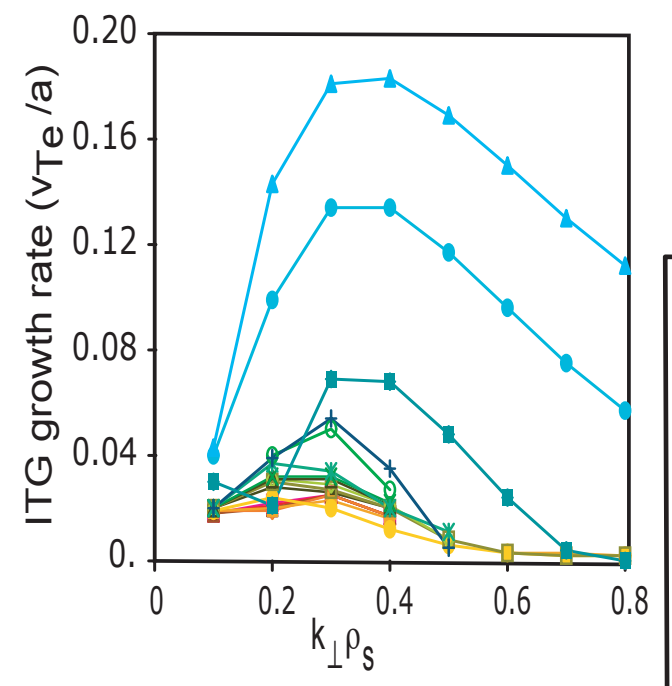

$\mathrm{IN} / \mathrm{N}$

Scaling factors

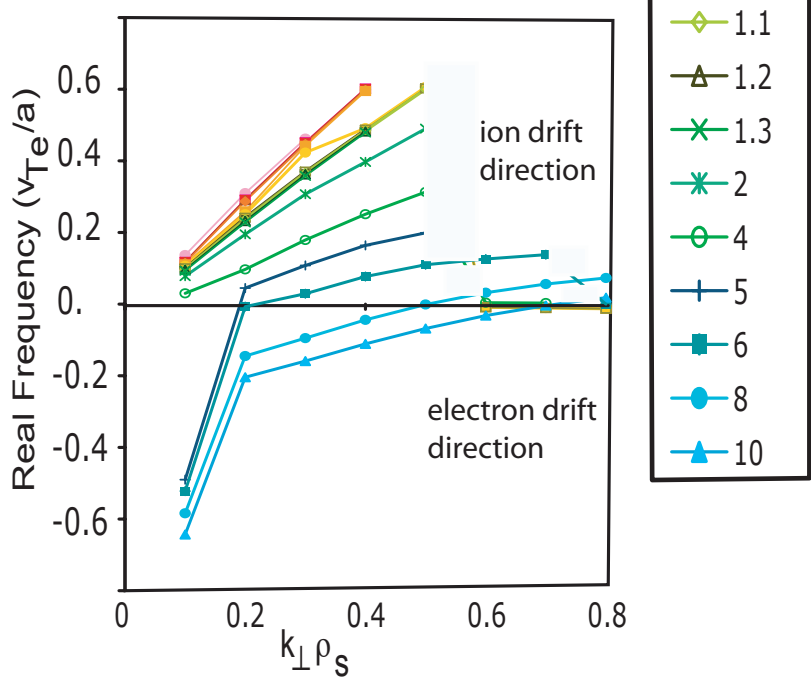

Fig 9 


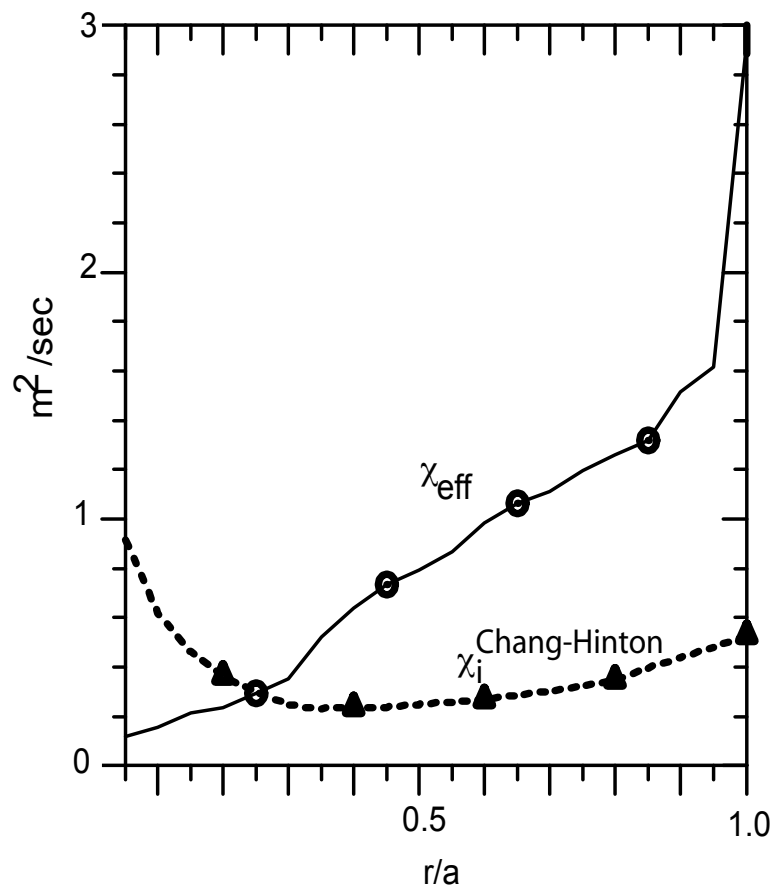

Fig. 10

Redi 


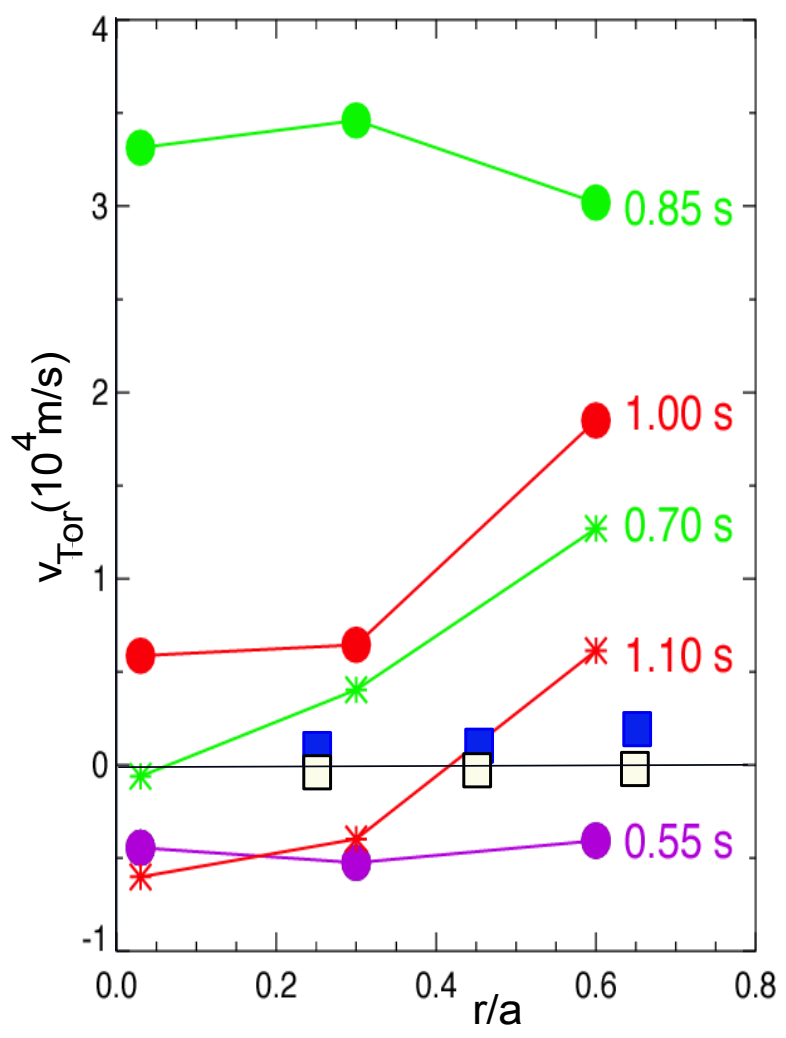

Fig 11

Redi 


\section{External Distribution}

Plasma Research Laboratory, Australian National University, Australia

Professor I.R. Jones, Flinders University, Australia

Professor João Canalle, Instituto de Fisica DEQ/IF - UERJ, Brazil

Mr. Gerson O. Ludwig, Instituto Nacional de Pesquisas, Brazil

Dr. P.H. Sakanaka, Instituto Fisica, Brazil

The Librarian, Culham Laboratory, England

Mrs. S.A. Hutchinson, JET Library, England

Professor M.N. Bussac, Ecole Polytechnique, France

Librarian, Max-Planck-Institut für Plasmaphysik, Germany

Jolan Moldvai, Reports Library, Hungarian Academy of Sciences, Central Research Institute for Physics, Hungary

Dr. P. Kaw, Institute for Plasma Research, India

Ms. P.J. Pathak, Librarian, Institute for Plasma Research, India

Ms. Clelia De Palo, Associazione EURATOM-ENEA, Italy

Dr. G. Grosso, Instituto di Fisica del Plasma, Italy

Librarian, Naka Fusion Research Establishment, JAERI, Japan

Library, Laboratory for Complex Energy Processes, Institute for Advanced Study, Kyoto University, Japan

Research Information Center, National Institute for Fusion Science, Japan

Dr. O. Mitarai, Kyushu Tokai University, Japan

Dr. Jiangang Li, Institute of Plasma Physics, Chinese Academy of Sciences, People's Republic of China

Professor Yuping Huo, School of Physical Science and Technology, People's Republic of China

Library, Academia Sinica, Institute of Plasma Physics, People's Republic of China

Librarian, Institute of Physics, Chinese Academy of Sciences, People's Republic of China

Dr. S. Mirnov, TRINITI, Troitsk, Russian Federation, Russia

Dr. V.S. Strelkov, Kurchatov Institute, Russian Federation, Russia

Professor Peter Lukac, Katedra Fyziky Plazmy MFF UK, Mlynska dolina F-2, Komenskeho Univerzita, SK-842 15 Bratislava, Slovakia

Dr. G.S. Lee, Korea Basic Science Institute, South Korea

Institute for Plasma Research, University of Maryland, USA

Librarian, Fusion Energy Division, Oak Ridge National Laboratory, USA

Librarian, Institute of Fusion Studies, University of Texas, USA

Librarian, Magnetic Fusion Program, Lawrence Livermore National Laboratory, USA

Library, General Atomics, USA

Plasma Physics Group, Fusion Energy Research Program, University of California at San Diego, USA

Plasma Physics Library, Columbia University, USA

Alkesh Punjabi, Center for Fusion Research and Training, Hampton University, USA

Dr. W.M. Stacey, Fusion Research Center, Georgia Institute of Technology, USA

Dr. John Willis, U.S. Department of Energy, Office of Fusion Energy Sciences, USA

Mr. Paul H. Wright, Indianapolis, Indiana, USA 
The Princeton Plasma Physics Laboratory is operated by Princeton University under contract with the U.S. Department of Energy.

\author{
Information Services \\ Princeton Plasma Physics Laboratory \\ P.O. Box 451 \\ Princeton, NJ 08543
}

Phone: 609-243-2750

Fax: 609-243-2751

e-mail: pppl_info@pppl.gov

Internet Address: http://www.pppl.gov 